\title{
Reconfigurable Intelligent Surface Based Orbital Angular Momentum: Architecture, Opportunities, and Challenges
}

\author{
Zhaohui Yang, Ye Hu, Zhaoyang Zhang, Wei Xu, Caijun Zhong, \\ Walid Saad, Fellow, IEEE, and Kai-Kit Wong, Fellow, IEEE
}

\begin{abstract}
Orbital angular momentum (OAM) has gained a lot attention due to its potential in enhancing spectral efficiency for wireless communications. Using different OAM modes, multiple independent data are simultaneously transmitted. OAM can bring a new multiple access for wireless communications. In this article, a reconfigurable intelligent surface (RIS) based OAM framework is introduced. The basic concepts and features of RIS and OAM are presented. Then, the classifications and comparisons of different RIS based OAM schemes are summarized. Furthermore, the RIS based OAM for wireless communications is discussed in details. Simulation results verify that the RIS based OMA transmission can significantly increase the spectral efficiency of wireless communication systems.
\end{abstract}

\section{INTRODUCTION}

Reconfigurable intelligent surfaces (RISs) are emerging technologies in the future sixth generation (6G) wireless communications for its great potential, such as the low power, low latency, high energy efficiency and massive connectivity [1]. RISs are mainly composed of large amounts of hardware efficient elements that can alter the phase of incoming signals, and such equipment necessitates a full-fledge communication infrastructure [2]. RIS-empowered system is capable of combating the unfavorable propagation environment. Unlike the conventional single antenna deployed at the relay node, the RIS with large number of elements enhances the quality of wireless communications. Specifically, the high frequency signal such as millimeter wave may be weakened during communications due to scattering effect or other unexpected channel conditions, however, the adoption of RIS technique can overcome these disadvantages by controlling the transmission environment. RIS-empowered system also provides an energy solution in wireless communications since the use of RIS reduces the hardware complexity, physical size, weight, and cost of conventional large arrays, thereby providing a more green communication architecture.

Orbital angular momentum (OAM) utilizes spatial distribution of helical phase front to generate a kind of new orthogonal resoure [3]. Different OAM modes are mutually orthogonal, allowing simultaneous and coaxial transmission to achieve higher spectral efficiency [4]. In order to generate OAM beams, spiral phase plates antenna, uniform circular array (UCA), and circular traveling-wave antenna are widely used in OAM multiplexing systems [5]-[7].

Theoretically, the number of single-beam orthogonal OAM modes is unlimited, and each mode is a fundamental element of complete orthogonality, which can be used to multiplex different signals, thereby greatly improving the spectral efficiency. It is shown in [8] that OAM represents the rotation of electrons around the propagation axis produced by the energy flow. Under certain conditions, OAM based communication can be obtained from the traditional multiple-input multipleoutput (MIMO) theory. However, beam divergence and misalignment will seriously reduce the transmission distance of OAM waves. Furthermore, moving and reflection will destroy the orthogonality of OAM waves, thereby reducing the performance under non-line-of-sight conditions.

The challenges faced by OAM and the advantages of RIS motivate this article to shift the focus of RIS based OAM design. The main contribution of this article is to introduce the RIS based OAM transmission framework. To introduce this new framework, we first provide a detailed overview on OAM and RIS, and summarize their advantages and challenges in Section II. The RIS based OAM with various classifications and challenges is introduced in Section III. Then, in Section IV, we introduce the implementation of RIS based OAM design for wireless communications, followed by the potential challenges and open problems. Conclusions are drawn in Section V.

\section{Preliminaries of OAM AND RIS}

In this section, we introduce the basic concepts and features of OAM and RIS.

\section{A. Basic Concept and Features of OAM}

OAM is considered as an orthogonal degree of freedom for data multiplexing [9]. A potential approach for simultaneously transmitting multiple data streams is to use a set of orthogonal electromagnetic (EM) waves. An EM wave with a helical transverse phase carries an OAM related to the integer, which is the number of azimuthal angles. As shown in Fig. 1(a), the OAM beam is generated by a UCA through feeding its antenna elements with the same input signal, but with a successive phase shift from element to element. The maximum number of OAM modes is the same as the number of elements in the UCA.

OAM multiplexing has the potential to increase the total transmission rate in wireless communication systems due to its ability to multiplex and simultaneously transmit multiple datacarrying channels on different OAM beams. As each OAM 

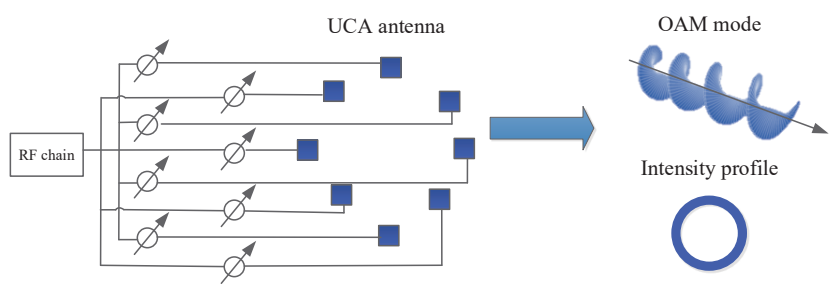

(a) The architecture of generating an OAM model

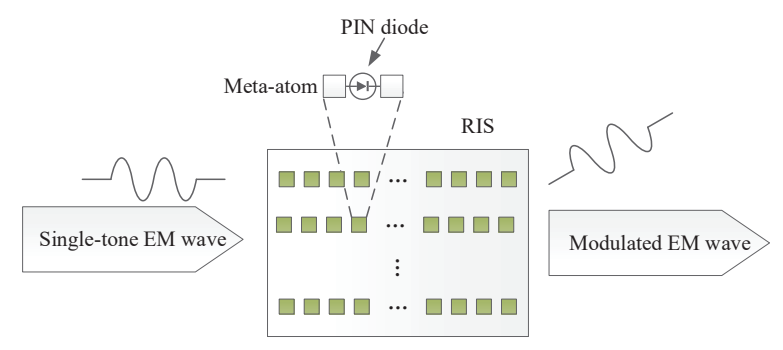

(b) The architecture of an RIS

Fig. 1. Architectures of (a) an OAM and (b) an RIS.

beam carrying an independent data stream co-propagates with other OAM beams, the spectral efficiency of the system (i.e., bits/s/Hz) scales with the number of OAM beams utilized for multiplexing. OAM beams can be carried on orthogonal polarizations and polarization division multiplexing (PDM) can also be applied to further double the spectral efficiency of the system [10].

\section{B. Basic Concept and Features of RIS}

RIS-assisted wireless communication has been proposed as a potential solution for enhancing the energy efficiency of wireless networks. An RIS is indeed a programmable metasurface equipped with low-cost and passive elements, which can manipulate EM waves. All RIS units are regularly arranged in a two-dimensional structure, as shown in Fig. 1(b) [11]. In RIS-assisted wireless communication networks, a base station (BS) sends control signals to an RIS controller so as to optimize the properties of incident waves and improve the communication quality of users. The RIS acts as a reflector and does not perform any digitalization operation. Hence, if properly deployed, an RIS promises much lower energy consumption than traditional amplify-and-forward (AF) relays. The constraint on the diagonal phase shift matrix and unit modulus of the reflecting RIS makes the design of RIS uniquely compared to the conventional wireless communication systems.

\section{Motivation of Implementing OAM with RIS}

OAM for wireless communication system has not been implemented as an independent information carrier for wireless holography, mainly due to the lack of OAM selectivity in conventional hologram design. The generation and detection of multiple wavefronts carrying the OAM have been demonstrated through holographic elements with only a few diffraction orders [10], [12]. Implementing OAM with high-resolution RIS could open up an unprecedented opportunity for ultrahighcapacity devices and systems due to a physically unbounded set of OAM modes.

\section{RIS BASED OAM}

RISs can program the incident EM signal by adjusting the reflection coefficients of the meta-atoms. This feature can be utilized to generate OAM beams. In this section, we elaborate on the idea of RIS based OAM via four schemes: centralized RIS (CRIS) based OAM with one transmit antenna, distributed RIS (DRIS) based OAM with one transmit antenna, CRIS based OAM with multiple transmit antennas, and DRIS based OAM with multiple transmit antennas. The corresponding system models are shown in Fig. 2.

\section{A. Classifications of RIS Based OAM}

1) CRIS based OAM with One Transmit Antenna: In CRIS based OAM with one transmit antenna, CRIS based OAM transmitter can directly perform modulations on the EM carrier signals, without the need for multiple conventional radiofrequency (RF) chains, which can be used for holographic multiuser MIMO technologies, as shown in Fig. 2(a). For the conventional RIS-relay communication systems, the doublefold channel gain is usually considered, i.e., effective channel gain between the BS and the user equals the channel gain between the BS and RIS times the the channel gain between the RIS and the user. In contrast, for the CRIS based OAM transmitter, we only need to consider the one-fold channel gain from the transmitter to users [13].

There are multiple transmit antennas in the traditional hybrid millimeter wave (mmWave) system, while only one transmit antenna is needed for the CRIS based OAM transmitter system in this case. For the traditional hybrid mmWave system, multiple RF chains are needed, while the proposed CRIS based OAM transmitter with one transmit antenna is considered as an RF chain-free transmitter.

2) DRIS based OAM with One Transmit Antenna: In particular, deploying multiple RISs in wireless networks has several advantages. First, distributed RISs can provide robust datatransmission since different DRISs can be deployed geometrically apart from each other. Meanwhile, multiple RISs can provide multiple paths of received signals, which increases the received signal strength.

Fig. 2(b) depicts the structure of DRIS based OAM with one transmit antenna. In this scheme, multiple RISs are deployed in a distributed manner and each RIS can turn the incident EM signal into one OAM mode.

3) CRIS based OAM with Multiple Transmit Antennas: Multiple antennas can transmit multiple data streams through using the spatial multiplexing. The combination of CRIS with multiple transmit antennas can allow transmitting multiple times of data streams than the CRIS with one transmit antenna. In particular, the total number of data streams for the CRIS based OAM with multiple transmit antennas is much larger than the number of transmit antenna since each antenna can transmit multiple OAM modes through CRIS as shown in Fig. 2(c). 


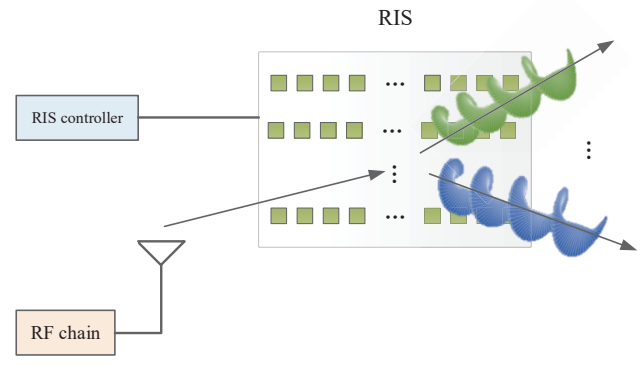

(a) CRIS based OAM with one transmit antenna

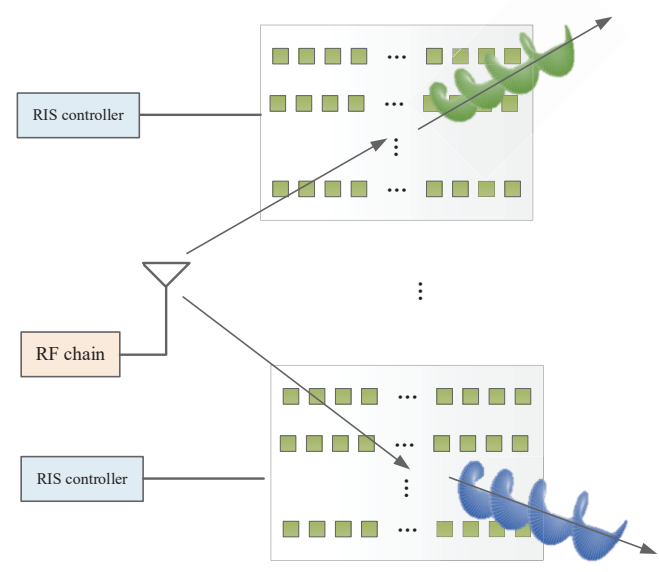

(b) DRIS based OAM with one transmit antenna

RIS

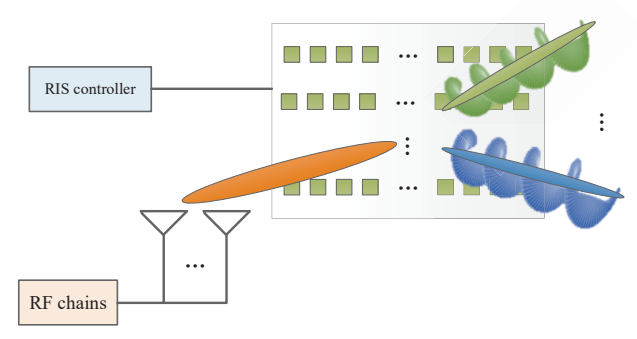

(c) CRIS based OAM with multiple transmit antennas

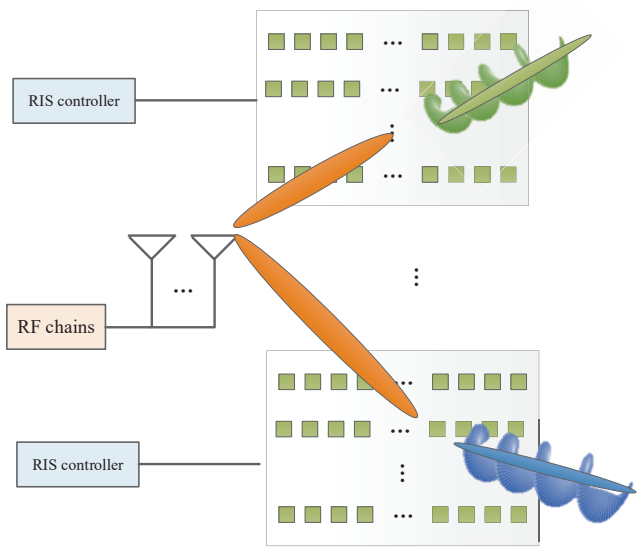

(d) DRIS based OAM with multiple transmit antennas

Fig. 2. RIS based OAM schemes: (a) CRIS based OAM with one transmit antenna, (b) DRIS based OAM with one transmit antenna, (c) CRIS based OAM with multiple transmit antennas, and (d) CRIS based OAM with multiple transmit antennas.
4) DRIS based OAM with Multiple Transmit Antennas: In DRIS based OAM scheme, each RIS can convert the incoming beamforming signal into an OAM mode based beamforming signal. Due to the orthogonality of different OAM beams, multiple beamforming signals can be transmitted at the same timefrequency resource in the DRIS based OAM with multiple transmit antennas, as shown in Fig. 2(d).

\section{B. Comparisons of RIS Based OAM}

Table I provides the considered RIS based OAM schemes in terms of number of RF chains, number of OAM modes, number of transmit antennas, spectral efficiency and power consumption. According to Table I, RIS based OAM is superior over antenna based OAM in terms of spectral efficiency and power consumption.

\section{RIS BASED OAM DESIGN FOR WIRELESS COMMUNICATIONS}

\section{A. Framework of RIS Based OAM for Wireless Communica- tions}

Due to high spectral efficiency and massive access, the RIS based OAM scheme is a potential solution for future wireless communication systems. An architecture of RIS based OAM design for wireless communications is shown in Fig. 3. At the transmitter side, one RF signal is required and an RIS controller determines the phase shift of all elements in the RIS to generate multiple OAM modes. Each mode can transmit one data stream and thus, multiple data streams can be transmitted at the same time and frequency resource. At the receiver side, the RIS is used to decouple the OAM modes. Since the modes are orthogonal, multiple data streams can be detected without any inter-mode interference.

As a case study, an RIS based OAM design for a wireless communication system is illustrated in Fig. 4. At the receiver side, there is one transmit antenna and one RIS with sixteen elements, while the receiver side is also equipped with an RIS with sixteen elements. In the transmission, quadrature phase shift keying (QPSK) modulation is adopted and two OAM beams are generated with controlling the phase element of the RIS at the receiver side. To compare the performance of RIS based OAM scheme (RIS-OAM), two baselines are considered: the conventional RIS scheme without any OAM beam as in [13] and the conventional single antenna transmission scheme without any RIS. Fig. 4 illustrates the sum rate of versus the transmit power of the antenna. It is shown that the rate of all schemes increases with the transmit power. According to Fig. 4, both RIS-OAM and conventional RIS schemes outperforms the conventional single antenna transmission scheme with no RIS. This is because the number of RIS elements is high and the strength of the received signal is high through the reflecting of the RIS, while there is no passive relay or OAM mode and the sum rate of the conventional single antenna transmission scheme with no RIS yields the lowest value. It is also observed that the OAM-RIS achieves the best sum rate, which indicates the superiority of using RIS to generate OAM modes. 
TABLE I

COMPARISONS OF ANTENNA BASED OAM WITH RIS BASED OAM.

\begin{tabular}{|c|c|c|c|c|c|c|}
\hline Class & Scheme & RF Chains & OAM Modes & $\begin{array}{l}\text { Transmit } \\
\text { Antennas }\end{array}$ & $\begin{array}{l}\text { Spectral Effi- } \\
\text { ciency }\end{array}$ & $\begin{array}{l}\text { Power Con- } \\
\text { sumption }\end{array}$ \\
\hline \multirow{2}{*}{ Antenna based OAM } & Centralized & High & Medium & High & Low & High \\
\hline & Distributed & High & Medium & High & Medium & High \\
\hline \multirow{4}{*}{ RIS based OAM } & $\begin{array}{l}\text { Centralized with } \\
\text { one antenna }\end{array}$ & 1 & Medium & 1 & Medium & Low \\
\hline & $\begin{array}{l}\text { Distributed with } \\
\text { one antenna }\end{array}$ & 1 & Medium & 1 & High & Low \\
\hline & $\begin{array}{l}\text { Centralized with } \\
\text { multiple antennas }\end{array}$ & Medium & High & Medium & High & Medium \\
\hline & $\begin{array}{l}\text { Distributed with } \\
\text { multiple antennas }\end{array}$ & Medium & High & Medium & Very High & Medium \\
\hline
\end{tabular}

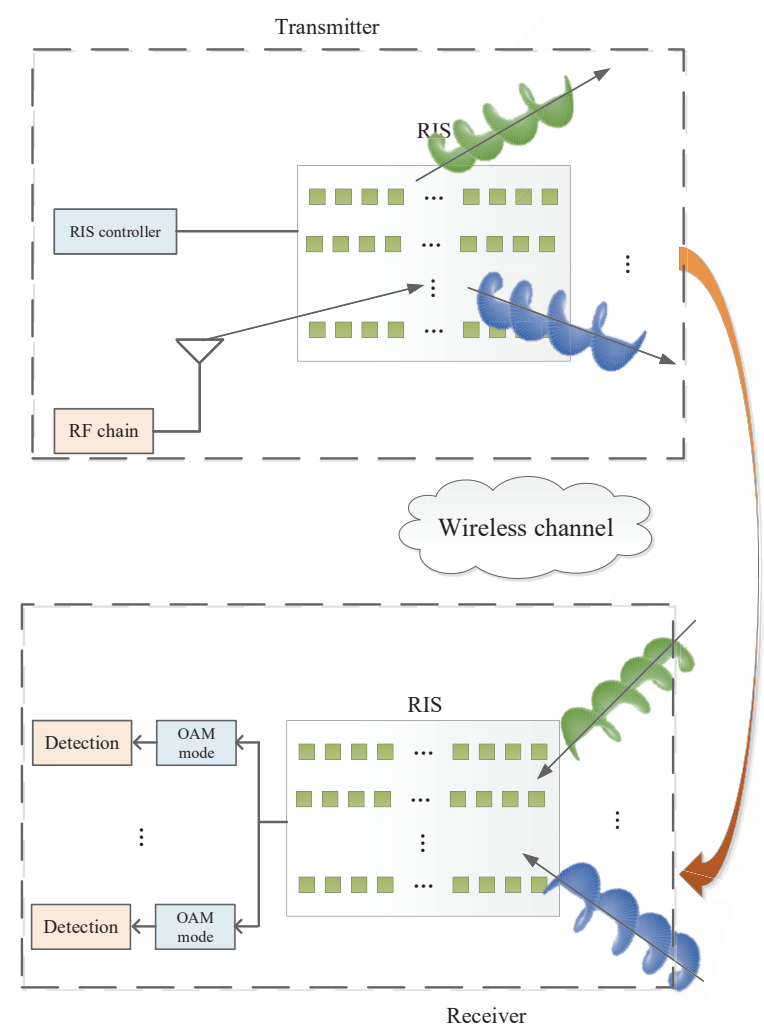

Fig. 3. An architecture of RIS based OAM design for wireless communications.

\section{B. Potential Challenges of RIS Based OAM Design for Wire- less Communications}

Despite the superiority of RIS based OAM scheme for wireless communications, there are some challenges in implementing RIS based OAM system for the wireless communication system.

1) Misalignment: The misalignment happens due to the fact the aperture of the receiver is not coaxial with the OAM modes from the RIS based OAM transmitter. In order to operate an OAM multiplex link, it is necessary to know which modes are being transmitted via RIS. The receiver aperture that captures the power around the center of the beam will recover the entire azimuthal phase change and know which

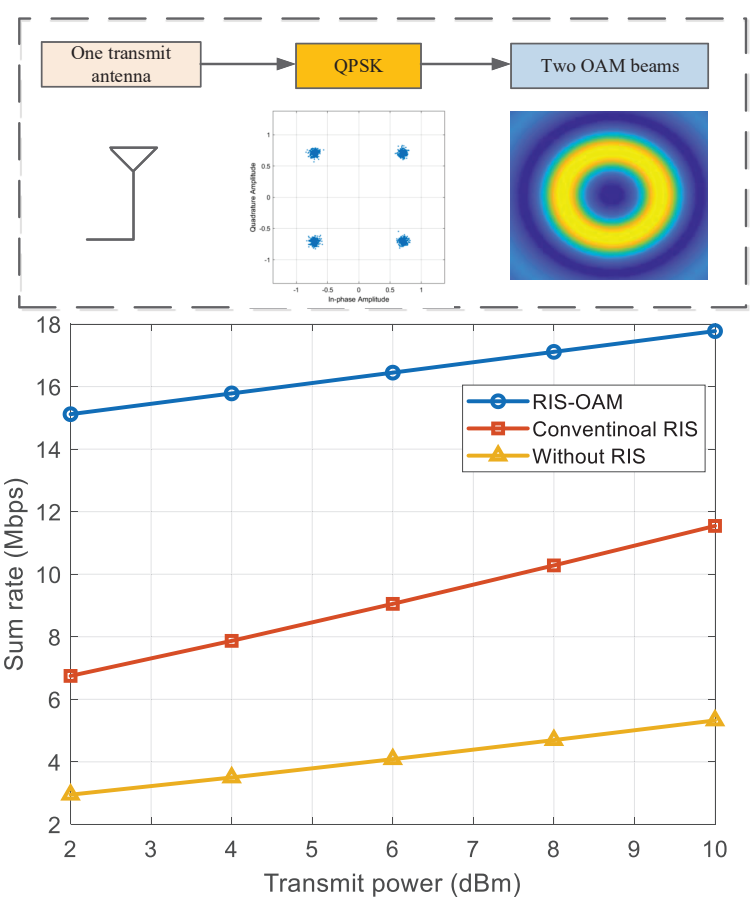

Fig. 4. Simulation scenario and result of RIS based OAM design for a wireless communication system.

mode was transmitted. However, an off-axis receiver aperture of restricted size will not be able to recover the complete phase change, and will inadvertently cannot identify different modes.

Effective multiplexing and demultiplexing of OAM beams requires coaxial transmission and reception of transmitted signals mode. Unlike the case of using Gaussian beams, any misalignment between the transmitter and receiver apertures or only partial collection of the OAM beam at the receiver will not only cause power loss, but more seriously cause crosstalk between channels. In an ideal OAM multiplexed communication link, the transmitter and receiver will be perfectly aligned. However, due to the jitter and vibration of the transmitter/receiver platform, the transmitter and receiver may have a relative lateral offset. Misalignment may cause system performance degradation.

2) Beam Divergence: The high-order OAM beam diverges faster than the low-order OAM beam, so it is difficult to fully capture the high-order OAM beam with a limited receiving 
aperture. If the beam power is not fully captured, power loss will occur, but due to the truncation of the beam's radial profile, even modal coupling will occur. This truncation may cause power to couple to other OAM beams generated by the RIS.

For communication links, it is better to collect as much signal power as possible at the receiver to ensure sufficient signal-to-noise ratio (SNR). According to diffraction theory, the OAM beam diverges when propagating in free space. Since circuit elements usually have finite-size apertures, the divergent light beam may be too large to be completely collected, resulting in signal power loss. For the OAM multiplex link, the transmission beam with a higher OAM order diverges faster than the beam with a lower OAM order. This makes it difficult to fully capture them with a limited size receiver aperture, resulting in signal power loss. In addition, the limited size of the receiver aperture also reduces orthogonality. The radial profile of the beam may be truncated, which may cause mode coupling from the desired mode to other OAM beams. The divergence effect of the OAM beam mainly depends on the frequency, the transmission distance, the beam waist at the transmitter and the mode index. Therefore, in order to reduce signal power loss, when designing RIS-based OAM links, it is necessary to carefully consider the key parameters related to beam divergence.

3) Channel Estimation: Due to the fact there are multiple OAM beams, the channel gains on different OAM beams are diverse. There are more channels to be estimated in RIS based OAM scheme than the conventional OAM or RIS scheme. In particular, the channel estimation in RIS based OAM system is different from that in traditional systems, since there are three kinds of channels: the channel between transmitter and RIS (transmitter-RIS channel), the channel between RIS and receiver (RIS-receiver channel), and the direct channel between transmitter and receiver. The direct channel can be estimated using conventional methods under the known cascaded channels, but how to obtain the cascaded channels (transmitter-RIS channel and RIS-receiver channel) is much complex. Besides, these three kinds of channels needs to be estimated for each OAM mode.

4) Resource Optimization: The resource allocation problem for RIS based OAM scheme involves the following variables: phase shift, OAM mode allocation, power control, and beamfroming design. For the phase shift, two kinds of models need to be considered, i.e., continuous phase and discrete phase. Due to different channel gains on different OAM mode, it is of importance to allocate the OAM mode to different users in the multiuser communication system. For the DRIS case, the pairing problem for OAM mode and RIS block should be considered. With given OAM mode selection, the power and beamforming needs to be jointly optimized by using the conventional optimization method. In particular, for the spectral efficiency optimization, the joint transmitter and receiver design can further improve the performance. For the energy efficiency optimization, although RIS is an passive surface, the energy consumption of the control signal for changing the phase shifter should be involved.

\section{Open Problems}

1) Frequency Division Duplex: Current methods all exploit the channel reciprocity of time division duplex (TDD) system, i.e., the uplink channel and downlink channel are assumed to be reciprocated, however, the investigation in frequency division duplex (FDD) system without channel reciprocity is insufficient. In addition, the high mobility system will lead to the high complexity in current methods. These two issues all require the RIS based OAM design to be robust to the environmental changes.

2) Imperfect Channel State Information: In the RIS based OAM system, to achieve the full advantages of the architecture, several efficient technologies are required including the joint active and passive beamforming, resource allocation, and energy-efficient design. It is noted that all of above designs rely on the perfect channel state information between the transmit antenna and RIS, and the perfect channel state information between the RIS and users. However, it is infeasible for the RIS based OAM systems to estimate the accurate channel state information when the RF chains or sensors are not equipped on the RIS.

3) Super Large RIS Design: The channel acquisition with the super large RISs has not been studied yet. The larger number of RISs would doubtless bring more benefits in wireless communication, but also involve more challenging issues. The training overhead of detection is significantly high, for example, model-based method requires numerous multiple training phases or training pilots and model-free methods has large offline data and higher hardware complexities to train model. Thus, how to efficiently estimate the involved channels for super large RISs based OAM scheme at low training cost is still open to investigate.

4) Joint Channel Estimation and Resource Allocation: The joint channel estimation and resource allocation can effectively improve the performance, however, it is still open to investigate for RIS based OAM scheme. The existing works in OAM or RIS system do not consider the joint design of channel estimation of RIS related channels over different OAM modes. Besides, the joint OAM mode allocation and phase shift design is still an open problem.

\section{Conclusions and Future Directions}

In this article, we have provided a new framework called RIS based OAM scheme to further increase the spectral efficiency of wireless communications. We have investigated four main classifications of RIS based OAM schemes. Besides, we have provided the challenges of the RIS based OAM schemes. Meanwhile, we have presented the RIS based OAM design for wireless communications. The discussed open opportunities identify necessary problems needed to be solved for implementing RIS based OAM scheme in wireless communications.

Zhaohui Yang is currently a Post-Doctoral Research Fellow at University College London, UK. His research interests include federated learning, reconfigurable intelligent surface, and unmanned aerial vehicles. 
Ye Hu is currently pursuing the Ph.D. degree with the Bradley Department of Electrical and Computer Engineering, Virginia Tech. Her research interests include game theory, cyber-physical systems, cybersecurity, unmanned aerial vehicles, and wireless communication.

Zhaoyang Zhang is currently a Qiushi Distinguished Professor at Zhejiang University. His research interests are mainly focused on next generation wireless communications, network intelligence, synergetic sensing-computationand-communication, etc.

Wei Xu is currently a Professor with the National Mobile Communications Research Laboratory, Southeast University. He has coauthored more than 100 refereed journal papers, 36 domestic patents, and four U.S. patents granted. His research interests include information theory, signal processing, and machine learning for wireless communications.

\section{REFERENCES}

[1] W. Saad, M. Bennis, and M. Chen, "A vision of 6G wireless systems: Applications, trends, technologies, and open research problems," IEEE network, vol. 34, no. 3, pp. 134-142, 2019.

[2] C. Huang, A. Zappone, G. C. Alexandropoulos, M. Debbah, and C. Yuen, "Reconfigurable intelligent surfaces for energy efficiency in wireless communication," IEEE Trans. Wireless Commun., vol. 18, no. 8, pp. 4157-4170, Aug. 2019.

[3] N. Bozinovic, Y. Yue, Y. Ren, M. Tur, P. Kristensen, H. Huang, A. E. Willner, and S. Ramachandran, "Terabit-scale orbital angular momentum mode division multiplexing in fibers," science, vol. 340, no. 6140, pp $1545-1548,2013$

[4] L. Allen, M. W. Beijersbergen, R. Spreeuw, and J. Woerdman, "Orbital angular momentum of light and the transformation of laguerre-gaussian laser modes," Physical review A, vol. 45, no. 11, p. 8185, 1992.

[5] Y. Yan, G. Xie, M. P. Lavery, H. Huang, N. Ahmed, C. Bao, Y. Ren, Y. Cao, L. Li, Z. Zhao et al., "High-capacity millimetre-wave communications with orbital angular momentum multiplexing," Nature Commun., vol. 5, no. 1, pp. 1-9, 2014.

[6] W. Zhang, S. Zheng, X. Hui, R. Dong, X. Jin, H. Chi, and X. Zhang, "Mode division multiplexing communication using microwave orbital angular momentum: An experimental study," IEEE Trans. Wireless Commun., vol. 16, no. 2, pp. 1308-1318, Feb. 2017

[7] X. Hui, S. Zheng, Y. Chen, Y. Hu, X. Jin, H. Chi, and X. Zhang, "Multiplexed millimeter wave communication with dual orbital angular momentum (OAM) mode antennas," Scientific Reports, vol. 5, no. 1, pp. $1-9,2015$.

[8] O. Edfors and A. J. Johansson, "Is orbital angular momentum (OAM) based radio communication an unexploited area?" IEEE Trans. Antennas Propagation, vol. 60, no. 2, pp. 1126-1131, 2011.

[9] W. Cheng, W. Zhang, H. Jing, S. Gao, and H. Zhang, "Orbital angular momentum for wireless communications," IEEE Wireless Commun., vol. 26, no. 1, pp. 100-107, 2018.

[10] A. E. Willner, Z. Zhao, C. Liu, R. Zhang, H. Song, K. Pang, K. Manukyan, H. Song, X. Su, G. Xie et al., "Perspectives on advances in high-capacity, free-space communications using multiplexing of orbital-angular-momentum beams," APL Photonics, vol. 6, no. 3, p 030901, 2021

[11] C. Huang, S. Hu, G. C. Alexandropoulos, A. Zappone, C. Yuen, R. Zhang, M. Di Renzo, and M. Debbah, "Holographic MIMO surfaces for $6 \mathrm{G}$ wireless networks: Opportunities, challenges, and trends," IEEE Wireless Commun., vol. 27, no. 5, pp. 118-125, 2020.

[12] H. Ren, G. Briere, X. Fang, P. Ni, R. Sawant, S. Héron, S. Chenot, S. Vézian, B. Damilano, V. Brändli et al., "Metasurface orbital angular momentum holography," Nature Commun., vol. 10, no. 1, pp. 1-8, 2019.

[13] Z. Yang, W. Xu, C. Huang, J. Shi, and M. Shikh-Bahaei, "Beamforming design for multiuser transmission through reconfigurable intelligent surface," IEEE Trans. Commun., 2020.
Walid Saad is is a full Professor at the Department of Electrical and Computer Engineering at Virginia Tech, where he leads the Network sciEnce, Wireless, and Security (NEWS@VT) laboratory, within the Wireless@VT research group. His research interests include wireless networks, machine learning, game theory, security, unmanned aerial vehicles, cyber-physical systems, smart grids, and network science.
Kai-Kit Wong is the Chair of Wireless Communications with the Department of Electronic and Electrical Engineering, University College London, London, U.K. His current research centers around $5 \mathrm{G}$ and beyond mobile communications. He is a Fellow of IET and is also on the editorial board of several international journals. He has been the Editor-in-Chief for IEEE Wireless Communications Letters since 2020. 